\title{
Influência da profundidade de polimerização, tempo e meio de armazenagem na dureza superficial de compósitos odontológicos convencionais e Bulk-Fill
}

Influence of depth of cure, time and storage means on the surface hardness of conventional and Bulk-Fill dental resin composites

Influencia de la profundidad de polimerización, el tiempo y los medios de almacenamiento en la dureza de la superficie de los compuestos dentales convencionais e Bulk-Fill

Ana Karina Maciel de Andrade

ORCID: https://orcid.org/0000-0003-4520-5176 Universidade Federal da Paraíba, Brasil E-mail: renallywanderley@gmail.com

Sônia Saeger Meireles

ORCID: https://orcid.org/0000-0001-7328-2991 Universidade Federal da Paraíba, Brasil

E-mail: soniasaeger@hotmail.com

Rosângela Marques Duarte

ORCID: https://orcid.org/0000-0003-4369-2951 Universidade Federal da Paraíba, Brasil E-mail: rose_marquesd@hotmail.com

\begin{abstract}
Resumo
O uso dos compósitos odontológicos tem crescido nos últimos anos devido ao aumento da demanda estética e surgimento de novas tecnologias. O grau de conversão está diretamente relacionado com o seu grau de dureza. Este estudo avaliou a influência da profundidade de polimerização, tempo e meio de armazenagem na dureza superficial de compósitos odontológicos. Três resinas compostas foram utilizadas: Filtek Z250 XT (3M/ESPE), Filtek Bulk Fill (3M/ESPE) e Tetric N-Ceram Bulk Fill (Ivoclar Vivadent). Foram confeccionados 20 corpos de prova para cada material, sendo 10 para cada meio de imersão (água e solução etanol/água $75 \%$ ) e 10 para cada profundidade de polimerização ( $0 \mathrm{~mm}, 2,0 \mathrm{~mm}$ e 4,0 $\mathrm{mm}$ ) para avaliação da dureza do topo, meio e base de cada corpo de prova. Para o ensaio da dureza inicial, em cada superfície foram realizadas três endentações com uma carga de 50 kgf, por 15 segundos em um microdurômetro. Em seguida os corpos de prova foram armazenados individualmente nos meios de armazenagem a $37^{\circ} \mathrm{C}$ pelos períodos de 24 horas, 90 dias e 180 dias. Todos os compósitos odontológicos avaliados apresentaram significativa redução da dureza com o aumento da profundidade de polimerização. Os compósitos demonstraram redução dos valores médios de dureza superficial ao longo do tempo, sendo mais pronunciado na armazenagem com solução de álcool. A microdureza de todas as resinas compostas reduziu com o aumento da profundidade de polimerização. O meio de armazenagem influenciou a microdureza superficial, sendo mais pronunciado para o meio de armazenagem solução de etanol a 75\%.
\end{abstract}

Palavras-chave: Dureza; Resinas compostas; Polimerização. 


\begin{abstract}
The use of dental composites has grown in recent years due to the increase in aesthetic demand and the emergence of new technologies. The degree of conversion is directly related to its degree of hardness. This study evaluated the influence of depth of cure, time and storage medium on the surface hardness of dental composites. Three composite resins were used: Filtek Z250 XT (3M/ESPE), Filtek Bulk Fill (3M/ESPE) and Tetric N-Ceram Bulk Fill (Ivoclar Vivadent). Twenty specimens were made for each material, 10 for each immersion medium (water and $75 \%$ ethanol/water solution) and 10 for each depth of cure $(0 \mathrm{~mm}, 2.0 \mathrm{~mm}$ and $4.0 \mathrm{~mm})$ for evaluation the hardness of the top, middle and base of each specimen. For the initial hardness test, three indentations were performed on each surface with a load of $50 \mathrm{kgf}$, for 15 seconds in a microdurometer. Then, the specimens were stored individually in storage media at $37^{\circ} \mathrm{C}$ for periods of 24 hours, 90 days and 180 days. All dental composites evaluated showed a significant reduction in hardness with increasing depth of cure. The composites showed a reduction in the average surface hardness values over time, being more pronounced in storage with an alcohol solution. The microhardness of all composite resins reduced with increasing depth of cure. The storage medium influenced the surface microhardness, being more pronounced for the storage medium, a $75 \%$ ethanol solution.
\end{abstract}

Keywords: Hardness; Composite resins; Polymerization.

\title{
Resumen
}

El uso de composites dentales ha crecido en los últimos años debido al aumento de la demanda estética y la aparición de nuevas tecnologías. El grado de conversión está directamente relacionado con su grado de dureza. Este estudio evaluó la influencia de la profundidad de polimerización, el tiempo y el medio de almacenamiento en la dureza de la superficie de los composites dentales. Se utilizaron tres resinas compuestas: Filtek Z250 XT (3M / ESPE), Filtek Bulk Fill (3M / ESPE) y Tetric N-Ceram Bulk Fill (Ivoclar Vivadent). Se realizaron veinte probetas para cada material, 10 para cada medio de inmersión (agua y solución de etanol / agua al 75\%) y 10 para cada profundidad de polimerización $(0 \mathrm{~mm}, 2.0 \mathrm{~mm}$ y $4.0 \mathrm{~mm})$ para evaluar la dureza de la parte superior, media y base. de cada espécimen. Para la prueba de dureza inicial, se realizaron tres hendiduras en cada superficie con una carga de $50 \mathrm{kgf}$, durante 15 segundos en un microdurómetro. Luego, las muestras se almacenaron individualmente en medios de almacenamiento a $37^{\circ} \mathrm{C}$ por períodos de 24 horas, 90 días y 180 días. Todos los composites dentales evaluados mostraron una reducción significativa de la dureza al aumentar la profundidad de polimerización. Los composites mostraron una reducción de los valores medios de dureza superficial a lo largo del tiempo, siendo más pronunciada en el almacenamiento con una solución alcohólica. La microdureza de todas las resinas compuestas se reduce al aumentar la profundidad de polimerización. El medio de almacenamiento influyó en la microdureza superficial, siendo más pronunciada para el medio de almacenamiento, una solución de etanol al $75 \%$.

Palabras clave: Dureza; Resinas compuestas; Polimerización.

\section{Introdução}

Devido à demanda estética, assim como ao avanço da tecnologia, um aperfeiçoamento das propriedades dos compósitos restauradores tem sido observado, favorecendo o aumento da qualidade das restaurações, e portanto, a longevidade dos procedimentos restauradores (Ribeiro et al., 2017).

Algumas propriedades como dureza e resistência mecânica são essenciais para um resultado clínico satisfatório em longo prazo. A dureza consiste na resistência de uma estrutura sólida à endentação permanente ou à penetração (Assis, 2015). Compósitos com baixa resistência à penetração são mais propensos a falhar, podendo comprometer a resistência do material à fadiga (Leite et al, 2018). A dureza é essencial para avaliar o desgaste do material, pois a baixa dureza pode indicar uma polimerização incompleta, causando alteração nas propriedades mecânicas dos compósitos, portanto é importante otimizar os métodos de conversão dos monômeros para se obter um melhor desempenho clínico desses materiais (El-Damanhoury \& Platt, 2014). Uma polimerização adequada vai aumentar as propriedades físicas do compósito, além de uma melhor adaptação marginal e uma redução na citotoxicidade causada pelos monômeros residuais (El-Safty et al., 2012).

O método convencional de inserção de compósitos na cavidade oral é a inserção por incrementos, que surgiu com a finalidade de diminuir as tensões oriundas da contração de polimerização e melhorar o selamento marginal das restaurações, mas há o risco da formação de espaços vazios entre as camadas, além do aumento de tempo de trabalho do cirurgião dentista (Campos et al., 2014). Outro fator a se considerar sobre os compósitos odontológicos convencionais é que quando inseridos em grandes incrementos podem causar manchamento, cáries marginas e sensibilidade pós-operatória decorrentes das tensões de contração de polimerização (Campos et al., 2014; Tiba et al., 2013). Nos últimos anos surgiram os compósitos do tipo Bulk- 
Fill, cuja recomendação do fabricante é da inserção em incremento único e profundidade de até 4,0mm ou 5,0mm (Bicalho et al., 2014; El-Safty et al., 2012; Ibarra et al., 2015). A profundidade de polimerização deve-se a maior translucidez, ao conteúdo e tamanho de partículas, além da adição de fotoiniciadores alternativos, que possuem maior absorção luminosa e controlam a cinemática da polimerização (Alshali et al., 2013; Fronza et al., 2015; Han et al., 2014).

Após a finalização da restauração, a mesma passa a interagir com flutuações de temperatura e pH do meio oral. Em adição, se o grau de conversão dessa restauração for reduzido poderá culminar, em longo prazo, na degradação hidrolítica do material no meio bucal, fazendo com que reduza as propriedades mecânicas, comprometendo a longevidade da restauração (Sunbul et al., 2016; Wei et al., 2011). A degradação hidrolítica do compósito é de extrema importância na seleção do compósito restaurador, pois o amolecimento da matriz resinosa pode prejudicar suas características, como a dureza e rugosidade superficial (Alshali et al., 2013). Portanto, é de extrema importância o estudo das características desses compósitos, visando uma maior durabilidade e excelência clínica.

Assim, esta pesquisa objetivou analisar a influência da profundidade de polimerização, tempo e meio de armazenamento na microdureza superficial de compósitos do tipo Bulk Fill e convencionais. A hipótese nula testada é de que a profundidade de polimerização, o tempo e os meios de armazenagem não influenciam a microdureza superficial dos compósitos.

\section{Metodologia}

O universo da pesquisa foi composto pelos compósitos Filtek Z250 XT (3M/ESPE), Filtek Bulk Fill (3M/ESPE) e Tetric N-Ceram Bulk Fill (Ivoclar Vivadent) (Figura 1). Informações sobre a composição e especificações dos materiais utilizados são encontrados na Tabela 1.

Figura 1. Resinas compostas utilizadas.

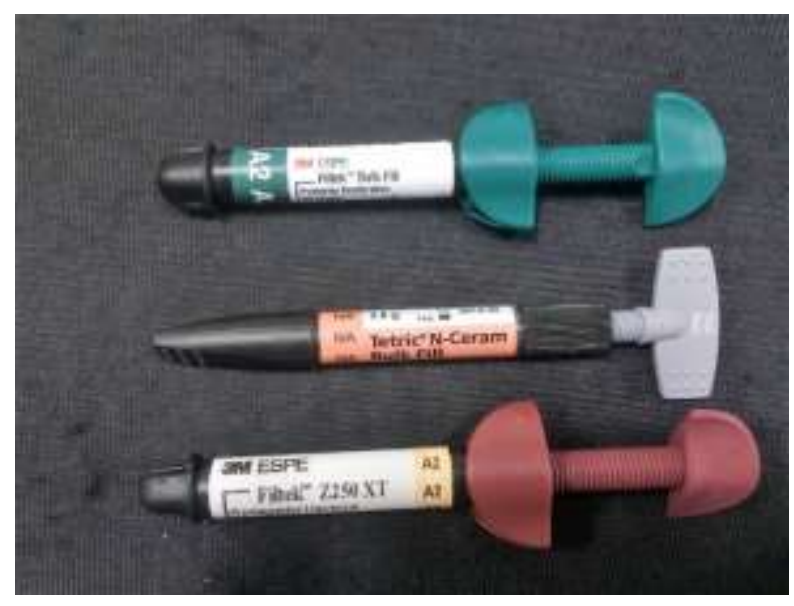

Fonte: Autores. 
Tabela 1. Composição da matriz orgânica, quantidade e tipo de partículas de carga dos compósitos estudados.

\begin{tabular}{|c|c|c|c|c|c|}
\hline Compósito & $\begin{array}{l}\text { Composição da } \\
\text { Matriz Orgânica }\end{array}$ & $\begin{array}{c}\text { Quantidade de } \\
\text { partículas de carga } \\
\text { (volume/peso) }\end{array}$ & Tipo de partículas de carga & Cor & Fabricante \\
\hline Filtek Z $250 \mathrm{XT}^{*}$ & $\begin{array}{c}\text { Bis-GMA, UDMA, } \\
\text { Bis-EMA-6, } \\
\text { TEGDMA, PEGDMA }\end{array}$ & $\begin{array}{c}67,8 \% \text { / } \\
81,8 \%\end{array}$ & $\begin{array}{c}\text { Combinação de sílica não } \\
\text { aglomeradas (20nm), partículas de } \\
\text { zircônia não aglomeradas ( } 3 \mathrm{~nm}) \text { e } \\
\text { aglomerado de zircônia/sílica } \\
\text { (combinação de sílica com } 20 \mathrm{~nm} \text { e } \\
\text { zircônia } 3 \mathrm{~nm}) .\end{array}$ & A2 & $3 \mathrm{M} / \mathrm{ESPE}$ \\
\hline Filtek Bulk Fill* & $\begin{array}{c}\text { AUDMA, UDMA e } \\
\text { 1, 12-dodecano- } \\
\text { DMA. }\end{array}$ & $\begin{array}{c}76,5 \% / \\
58,5 \%\end{array}$ & $\begin{array}{l}\text { Combinação de partículas de sílica de } \\
20 \mathrm{~nm} \text { não-aglomeradas, partículas de } \\
\text { zircônia de } 4 \text { a } 11 \mathrm{~nm} \text { não- } \\
\text { aglomeradas, nanoaglomerados de } \\
\text { zircônia/sílica (partículas de sílica de } \\
20 \mathrm{~nm} \text { e partículas de zircônia de } 4 \text { a } \\
11 \mathrm{~nm} \text { ) e partículas de trifluoreto de } \\
\text { itérbio em partículas aglomeradas de } \\
100 \mathrm{~nm}\end{array}$ & $\mathrm{~A} 2$ & $3 \mathrm{M} / \mathrm{ESPE}$ \\
\hline $\begin{array}{l}\text { Tetric N-Ceram Bulk } \\
\text { Fill* }\end{array}$ & $\begin{array}{l}\text { Bis-GMA, UDMA, } \\
\text { Bis-EMA }\end{array}$ & $\begin{array}{c}53-55 \% / \\
75-77 \%\end{array}$ & $\begin{array}{l}\text { Vidrio de bário, pré-polímero, tri- } \\
\text { fluoreto de itérbio e óxidos mistos. }\end{array}$ & IVA & $\begin{array}{c}\text { Ivoclar } \\
\text { Vivadent }\end{array}$ \\
\hline
\end{tabular}

Bis-GMA: Bisfenol A glicidilmetacrilato

Bis-EMA-6: Bisfenol-A polietilenoflicol diéter dimetacrilato

UDMA: Uretano dimetacrilato

TEGDMA: trietilenoglicol dimetacrilato

PEGDMA: polietilenoglicol dimetacrilato

AUDMA: Uretano Dimetacrilato Aromático

Fonte: Perfil técnico do fabricante*

Foram confeccionados 20 corpos de prova para cada material utilizando duas matrizes com cavidade central de 5,0 mm de diâmetro e espessura de $2,0 \mathrm{~mm}$, sendo 10 corpos de prova para cada meio de imersão (água destilada e solução etanol $75 \%$ ) e profundidade de polimerização de $0,0 \mathrm{~mm}, 2,0 \mathrm{~mm}$ e $4,0 \mathrm{~mm}$. Sobre uma placa de vidro foi posicionada uma tira de poliéster e sobre esta a primeira matriz. Em seguida, o compósito foi inserido e uma nova tira de poliéster foi posicionada sobre o mesmo. Sobre o conjunto, foi posicionada uma segunda matriz para inserção de nova porção do compósito e posicionamento de última tira de poliéster. A fotopolimerização do conjunto de matrizes foi realizada de acordo com o tempo recomendado por cada fabricante da resina (Figura 2). Após polimerização os corpos de prova foram retirados das matrizes e foi realizado a remoção dos excessos de compósito com lâmina de bisturi. A superfície de topo e base foram marcadas, e, posteriormente imersas em água destilada/deionizada por 24 horas a $37^{\circ} \mathrm{C}$, em estufa. 
Figura 2. Desenho ilustrando a confecção dos corpos de prova: camadas de resina inseridas (B) em moldes de teflon (A) sobrepostos e separados por fita de poliéster e lâminas de vidro $(C)$ e fotoativação efetuada no último incremento formando um cilindro com dois discos.
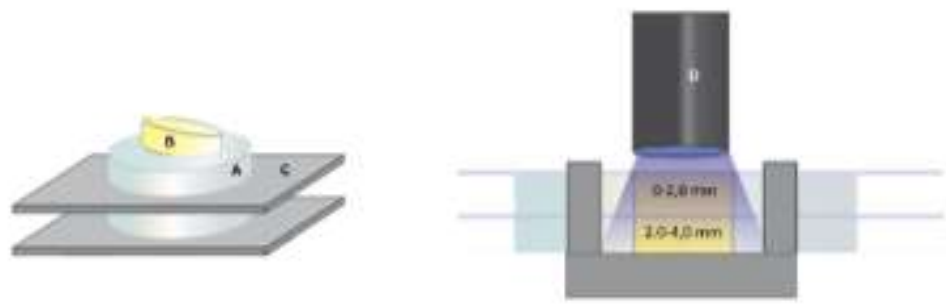

Fonte: Autores.

Para o ensaio de microdureza inicial, a leitura foi realizada no topo do corpo de prova obtido pela inserção da resina na segunda matriz, correspondendo a $0,0 \mathrm{~mm}$ de profundidade. Para calcular a dureza nas outras três profundidades $(0,0,2,0$ e $4 \mathrm{~mm}$ ). Em cada superfície, foram realizadas três endentações com uma carga de 50 kgf, por 15 segundos no microdurômetro HMV-G20 (Shimadzu). Em seguida, os corpos de prova foram armazenados individualmente nos meios de armazenagem a $37^{\circ} \mathrm{C}$ pelos períodos de 24 horas, 90 dias e 180 dias. Decorrido esses períodos de armazenagem foi realizado o ensaio de microdureza nas condições de teste descritas acima.

Os valores médios de microdureza dos materiais nas condições de teste avaliados foram submetidos aos testes estatísticos Kruskal-Wallis, Mann-Whitney para comparar compósitos diferentes e Friedman e Wilcoxon para comparar o mesmo compósito em diferentes profundidades e tempo de avaliação. O programa estatístico foi SPSS versão 20.

\section{Resultados}

O compósito Filtek Z250, no meio de imersão água destilada (Tabela 2), apresenta maiores valores de dureza, quando comparados ao compósito Tetric N-Ceram e Filtek Bulk Fill, exceto na profundidade de 4 mm, em 24 horas, quando a dureza do compósito Filtek Bulk Fill é superior a da Filtek Z250, já em 90 dias, na profundidade de 4 mm, não há diferença estatística significativa entre a Filtek Z250 e a Filtek Bulk Fill.

Tabela 2. Valores médios de microdureza nas profundidades de 0,$0 ; 2,0$ e 4,0 $\mathrm{mm}$ entre materiais, por profundidade e por meio de armazenagem após período de 24 horas, 90 dias e 180 dias de armazenagem em água destilada.

\begin{tabular}{|l|c|c|c|c|c|c|c|c|c|}
\cline { 2 - 9 } \multicolumn{1}{c|}{} & \multicolumn{9}{c|}{ ÁGUA DESTILADA } \\
\cline { 2 - 10 } \multicolumn{1}{c|}{} & \multicolumn{3}{c|}{24 HORAS } & \multicolumn{3}{c|}{90 DIAS } & \multicolumn{3}{c|}{180 DIAS } \\
\hline Material & $0,0 \mathrm{~mm}$ & $2,0 \mathrm{~mm}$ & $4,0 \mathrm{~mm}$ & $0,0 \mathrm{~mm}$ & $2,0 \mathrm{~mm}$ & $4,0 \mathrm{~mm}$ & $0,0 \mathrm{~mm}$ & $2,0 \mathrm{~mm}$ & $4,0 \mathrm{~mm}$ \\
\hline Z250 & $83,88 \pm 6,01 \mathrm{a}$ & $83,88 \pm 5,67 \mathrm{a}$ & $60,92 \pm 2,60 \mathrm{~b}$ & $83,68 \pm 2,99 \mathrm{a}$ & $83,82 \pm 4,14 \mathrm{a}$ & $62,76 \pm 4,21 \mathrm{a}$ & $90,92 \pm 1,98 \mathrm{a}$ & $88,57 \pm 1,08 \mathrm{a}$ & $69,50 \pm 5,01 \mathrm{a}$ \\
\hline TETRIC & $48,20 \pm 2,11 \mathrm{c}$ & $48,58 \pm 4,05 \mathrm{c}$ & $41,66 \pm 1,79 \mathrm{c}$ & $59,30 \pm 3,23 \mathrm{c}$ & $53,40 \pm 4,52 \mathrm{c}$ & $48,82 \pm 0,72 \mathrm{~b}$ & $58,98 \pm 5,35 \mathrm{c}$ & $50,98 \pm 5,02 \mathrm{c}$ & $47,72 \pm 2,25 \mathrm{~b}$ \\
\hline FILTEK & $66,29 \pm 4,94 \mathrm{~b}$ & $70,12 \pm 7.90 \mathrm{~b}$ & $66,22 \pm 3,56 \mathrm{a}$ & $68,66 \pm 4,48 \mathrm{~b}$ & $70,92 \pm 6,29 \mathrm{~b}$ & $63,54 \pm 4,80 \mathrm{a}$ & $72,42 \pm 4,11 \mathrm{~b}$ & $68,92 \pm 2,06 \mathrm{~b}$ & $65,13 \pm 4,35 \mathrm{a}$ \\
\hline
\end{tabular}

* Médias seguidas da mesma letra minúscula, nas colunas, não diferem estatisticamente entre si ao nível de 5\% de significância.

Fonte: Autores. 
Ainda no mesmo meio, o compósito Tetric N-Ceram apresentou menor dureza quando comparado aos compósitos Filtek Bulk Fill e Filtek Z250, em todos os períodos de tempo e em todas as profundidades.

Já o compósito Filtek Bulk Fill apresentou valores intermediários quando comparados a Filtek Z250 e Tetric NCeram, exceto em 24 horas, na profundidade de 4 mm, pois apresentou maior dureza que a Filtek Z250 e em 90 dias, na profundidade de 4 mm não apresenta diferença estatística significativa quando comparada a Filtek Z250.

No meio de imersão Etanol 75\% (Tabela 3), o compósito Filtek Z250 apresenta melhores valores de dureza, quando comparados aos outros compósitos analisados, exceto em 24 horas, na profundidade de 4 mm, onde o compósito Filtek Bulk Fill não apresentou diferença significativa no valor de dureza, quando comparados a Filtek Z250.

Tabela 3. Valores médios de microdureza nas profundidades de 0,$0 ; 2,0$ e 4,0 $\mathrm{mm}$ entre materiais, por profundidade e por meio de armazenagem após período de 24horas, 90 dias e 180 dias de armazenagem em etanol 75\%.

\begin{tabular}{|c|c|c|c|c|c|c|c|c|c|}
\hline \multirow{3}{*}{ Material } & \multicolumn{9}{|c|}{ ETANOL 75\% } \\
\hline & \multicolumn{3}{|c|}{24 HORAS } & \multicolumn{3}{|c|}{90 DIAS } & \multicolumn{3}{|c|}{180 DIAS } \\
\hline & $0,0 \mathrm{~mm}$ & $2,0 \mathrm{~mm}$ & $4,0 \mathrm{~mm}$ & $0,0 \mathrm{~mm}$ & $2,0 \mathrm{~mm}$ & $4,0 \mathrm{~mm}$ & $0,0 \mathrm{~mm}$ & $2,0 \mathrm{~mm}$ & $4,0 \mathrm{~mm}$ \\
\hline Z250 & $83,46 \pm 2,21 \mathrm{a}$ & $80,99 \pm 3,76 \mathrm{a}$ & $51,03 \pm 3,80 \mathrm{a}$ & $79,40 \pm 1,60 \mathrm{a}$ & $78,00 \pm 7,68 \mathrm{a}$ & $63,28 \pm 7,34 \mathrm{a}$ & $76,19 \pm 1,77 \mathrm{a}$ & $70,30 \pm 2,56 \mathrm{a}$ & $61,14 \pm 8,31 \mathrm{a}$ \\
\hline TETRIC & $32,96 \pm 2,09 \mathrm{c}$ & $30,44 \pm 2,01 \mathrm{c}$ & $26,41 \pm 1,9 b$ & $38,52 \pm 6,71 \mathrm{c}$ & $39,22 \pm 8,41 \mathrm{c}$ & $36,98 \pm 8,54 \mathrm{c}$ & $38,65 \pm 3,23 \mathrm{c}$ & $35,97 \pm 5,86 \mathrm{c}$ & $33,62 \pm 4,83 \mathrm{c}$ \\
\hline FILTEK & $65,39 \pm 5,15 b$ & $60,55 \pm 4,01 \mathrm{~b}$ & $51,58 \pm 6,51 \mathrm{a}$ & $55,32 \pm 5,79 b$ & $57,20 \pm 3,80 \mathrm{~b}$ & $49,08 \pm 2,58 b$ & $58,76 \pm 2,90 \mathrm{~b}$ & $53,90 \pm 2,81 \mathrm{~b}$ & $47,55 \pm 2,63 b$ \\
\hline
\end{tabular}

* Médias seguidas da mesma letra minúscula nas colunas não diferem estatisticamente entre si ao nível de 5\% de significância. Fonte: Autores

No meio de imersão Etanol 75\% (Tabela 3), o compósito Filtek Z250 apresenta melhores valores de dureza, quando comparados aos outros compósitos analisados, exceto em 24 horas, na profundidade de 4 mm, onde o compósito Filtek Bulk Fill não apresentou diferença significativa no valor de dureza, quando comparados a Filtek Z250.

O compósito Tetric N-Ceram, apresenta menores valores de dureza, quando comparados aos outros compósitos analisados, em todas as profundidades e em todos os períodos de tempo.

Ainda no mesmo meio de imersão, o compósito Filtek Bulk Fill apresenta valores intermediários quando comparados aos compósitos Tetric N-Ceram e Filtek Z250, exceto na profundidade de $4 \mathrm{~mm}$, em 24 horas, quando não apresenta diferença significativa de dureza, em relação a Filtek Z250.

O compósito Filtek Z250, no meio de imersão água destilada (Tabela 4), não apresenta diferenças significativas de dureza, nas profundidades de $0 \mathrm{~mm}$ e $2 \mathrm{~mm}$, em todos os períodos de tempo analisados, mas quando analisamos a profundidade de $4 \mathrm{~mm}$ há uma diminuição significativa na dureza, em todos os períodos de tempo. Não houve diferença significativa da dureza com o decorrer do tempo para esse compósito. 
Tabela 4. Valores médios de dureza nas profundidades de 0,0, 2,0 e 4,0 $\mathrm{mm}$ por material no meio de imersão água destilada após o período de 24 horas, 90 dias e 180 dias de armazenagem.

\begin{tabular}{|c|c|c|c|c|c|c|c|c|c|}
\hline & \multicolumn{9}{|c|}{ Água destilada } \\
\hline & \multicolumn{3}{|c|}{ Filtek Z250 } & \multicolumn{3}{|c|}{ Filtek Bulk Fill } & \multicolumn{3}{|c|}{ Tetric N-Ceram } \\
\hline & 24 horas & 90 dias & 180 dias & 24 horas & 90 dias & 180 dias & 24 horas & 90 dias & 180 dias \\
\hline $0 \mathrm{~mm}$ & $83,88 \pm 6,01 \mathrm{aA}$ & $83,68 \pm 2,99 \mathrm{aA}$ & $90,92 \pm 1,98 \mathrm{aA}$ & $66,29 \pm 4,94 \mathrm{aA}$ & $68,66 \pm 4,48 \mathrm{aA}$ & $72,42 \pm 4,11 \mathrm{aA}$ & $48,20 \pm 2,11 \mathrm{aB}$ & $59,30 \pm 3,23 \mathrm{aA}$ & $58,98 \pm 5,35 \mathrm{aA}$ \\
\hline $2 \mathrm{~mm}$ & $83,88 \pm 5,67 \mathrm{aA}$ & $83,82 \pm 4,14 \mathrm{aA}$ & $88,57 \pm 1,08 \mathrm{aA}$ & $70,12 \pm 7.90 \mathrm{aA}$ & $70,92 \pm 6,29 \mathrm{aA}$ & $68,92 \pm 2,06 \mathrm{aA}$ & $48,58 \pm 4,05 \mathrm{aA}$ & $53,40 \pm 4,52 \mathrm{abB}$ & $50,98 \pm 5,02 \mathrm{aB}$ \\
\hline $4 \mathrm{~mm}$ & $66,22 \pm 3,56 \mathrm{bA}$ & $62,76 \pm 4,21 \mathrm{bA}$ & $69,50 \pm 5,01 \mathrm{bA}$ & $66,22 \pm 3,56 \mathrm{aA}$ & $63,54 \pm 4,80 \mathrm{aA}$ & $65,13 \pm 4,35 \mathrm{aA}$ & $41,66 \pm 1,79 \mathrm{bA}$ & $48,82 \pm 0,72 \mathrm{bB}$ & $47,72 \pm 2,25 \mathrm{aB}$ \\
\hline
\end{tabular}

*Médias seguidas da mesma letra minúscula nas colunas e maiúsculas das linhas não diferem estatisticamente entre si ao nível de 5\% de significância.

Fonte: Autores.

No compósito Filtek Bulk Fill, no meio de imersão água destilada (Tabela 4), não houve diferença significativa na dureza, nas profundidades de $0 \mathrm{~mm}, 2 \mathrm{~mm}$ e $4 \mathrm{~mm}$. Ainda nesse mesmo compósito, não foi observado diferença significativa do valor de dureza com o decorrer do tempo, em todas as profundidades.

Já no compósito Tetric N-Ceram, no meio de imersão água destilada (Tabela 4), em 24 horas, houve diminuição significativa da dureza, na profundidade de 4 mm, já em 90 dias, essa diminuição foi observada na profundidade 4 mm. No decorrer do tempo, em todas as profundidades, houve aumento significativo da dureza nos períodos de 90 dias, não apresentando diferença estatística no período de 180 dias.

No compósito Filtek Z250, no meio de imersão Etanol 75\% (Tabela 5), houve diminuição significativa da dureza, nas profundidades de 4 mm em 24 horas, já em 180 dias, houve diminuição significativa em todas as profundidades analisadas, sendo a menor dureza em $4 \mathrm{~mm}$. Com o decorrer do tempo, não houve diferença significativa nos valores de microdureza em todas as profundidades.

Tabela 5 - Valores médios de dureza nas profundidades de 0,0, 2,0 e 4,0 mm por material no meio de imersão etanol a 75\% após o período de 24 horas, 90 dias e 180 dias de armazenagem.

\begin{tabular}{|c|c|c|c|c|c|c|c|c|c|}
\hline & \multicolumn{9}{|c|}{ Etanol a 75\% } \\
\hline & \multicolumn{3}{|c|}{ Filtek Z250 } & \multicolumn{3}{|c|}{ Filtek Bulk Fill } & \multicolumn{3}{|c|}{ Tetric N-Ceram } \\
\hline & 24 horas & 90 dias & 180 dias & 24 horas & 90 dias & 180 dias & 24 horas & 90 dias & 180 dias \\
\hline $0 \mathrm{~mm}$ & $76,19 \pm 1,77 \mathrm{aA}$ & $79,40 \pm 1,60 \mathrm{aA}$ & $76,19 \pm 1,77 \mathrm{aA}$ & $65,39 \pm 5,15 \mathrm{aA}$ & $55,32 \pm 5,79 \mathrm{aA}$ & $58,76 \pm 2,90 \mathrm{aA}$ & $47,55 \pm 2,63 \mathrm{aA}$ & $38,52 \pm 6,71 \mathrm{aA}$ & $38,65 \pm 3,23 \mathrm{aA}$ \\
\hline $2 \mathrm{~mm}$ & $80,99 \pm 3,76 \mathrm{aA}$ & $78,00 \pm 7,68 \mathrm{aA}$ & $70,30 \pm 2,56 \mathrm{bA}$ & $60,55 \pm 4,01 \mathrm{aA}$ & $57,20 \pm 3,80 \mathrm{aA}$ & $53,90 \pm 2,81 \mathrm{bB}$ & $30,44 \pm 2,01 \mathrm{bA}$ & $39,22 \pm 8,41 \mathrm{aA}$ & $35,97 \pm 5,86 \mathrm{aA}$ \\
\hline $4 \mathrm{~mm}$ & $51,58 \pm 6,51 \mathrm{bA}$ & $63,28 \pm 7,34 \mathrm{aA}$ & $61,14 \pm 8,31 \mathrm{cA}$ & $51,58 \pm 6,51 \mathrm{bA}$ & $49,08 \pm 2,58 \mathrm{bA}$ & $47,55 \pm 2,63 \mathrm{cA}$ & $26,41 \pm 1,91 \mathrm{cB}$ & $36,98 \pm 8,54 \mathrm{aA}$ & $33,62 \pm 4,83 \mathrm{aA}$ \\
\hline
\end{tabular}

* Médias seguidas da mesma letra minúscula nas colunas e maiúsculas das linhas não diferem estatisticamente entre si ao nível de 5\% de significância.

Fonte: Autores.

No compósito Filtek Bulk Fill, no meio de imersão Etanol 75\% (Tabela 5), nas profundidades de $0 \mathrm{~mm}$ e $2 \mathrm{~mm}$, houve diminuição significativa da dureza nos períodos de 24 horas e 90 dias, já em 180 dias essa diminuição foi observada em todas as profundidades analisadas. Com o decorrer do tempo, foi observada diminuição da dureza apenas no período de 180 dias, na profundidade de $2 \mathrm{~mm}$.

No compósito Tetric N-Ceram, no meio de imersão Etanol 75\% (Tabela 5), em 24 horas houve diminuição significativa na profundidade de $4 \mathrm{~mm}$, entretanto nos períodos de 90 dias e 180 dias, não houve diferença significativa com o aumento da profundidade. Com o decorrer do tempo, apenas em $4 \mathrm{~mm}$, houve um aumento significativo da dureza nos períodos de 90 dias e 180 dias. 


\section{Discussão}

Para avaliar o grau de polimerização, o ensaio de dureza superficial tem sido um método prático que fornece bons resultados (Alrahlah et al., 2014; Fronza et al., 2015; Yap et al., 2016). O teste de microdureza Vickers é o mais indicado para compósitos odontológicos devido sua maior estabilidade (Silveira et al., 2012).

Para uma polimerização eficiente, são fundamentais adequados comprimentos de onda e tempo de exposição durante a fotoativação. Também deve-se considerar que outros fatores afetam a profundidade de polimerização, incluindo tipo de compósito, cor e translucidez, espessura de incremento e a distância entre a ponta do aparelho fotoativador e a resina composta. Dentre esses fatores, o conteúdo, tamanho e distribuição de partículas são essenciais ao espalhamento do feixe de luz (Alrahlah et al., 2014; Fronza et al., 2015; Han et al., 2014).

A Food and Drug Admnistration (FDA) dos Estados Unidos indica a imersão em solução de etanol e água a 75\% como alternativa que simula as condições presentes na cavidade bucal (Sunbul et al., 2016). O meio e o período de armazenagem influenciaram diretamente na taxa de absorção e sorção e solubilidade. Soluções a base de etanol aceleram a penetração deste na matriz orgânica, com consequente liberação de componentes não reagidos. A água também exerce esse efeito, entretanto com menor rapidez (Ferracane, 2011).

Nas Tabelas de 2 a 5 observamos a comparação entre os valores médios de microdureza superficial entre materiais, para cada profundidade de polimerização, avaliados nesse estudo $(0,2$ e $4 \mathrm{~mm})$, e nos períodos de armazenagem. A hipótese nula testada foi rejeitada já que a profundidade de polimerização, o tempo e os meios de armazenagem influenciam a microdureza superficial dos compósitos estudados. O compósito Filtek Z250, no meio de imersão água destilada (Tabela 2), apresentou os maiores valores médios de dureza quando comparado aos compósitos Tetric N-Ceram e Filtek Bulk Fill, exceto nos períodos de armazenagem de 90 e 180 dias na profundidade de $4 \mathrm{~mm}$, onde não houve diferença estatística entre o compósito Filtek Z250 e Filtek Bulk Fill. No período de 24 horas, na profundidade de 4 mm, o compósito Filtek Bulk Fill apresentou maior dureza, com diferença estatística, quando comparado a Filtek Z250. No meio de imersão Etanol 75\% (Tabela 3), o compósito Filtek Z250 apresentou maior valor de dureza, quando comparados aos compósitos Tetric N-Ceram e Filtek Bulk Fill, exceto no período de 24 horas, na profundidade de $4 \mathrm{~mm}$, onde não houve diferença estatística entre o compósito Filtek Z250 e Filtek Bulk Fill.

A redução dos valores médios de dureza na profundidade de $4 \mathrm{~mm}$ pode ser explicada devido ao compósito ser convencional, ou seja, necessita ser inserido em incrementos de, no máximo, $2 \mathrm{~mm}$ para ter um grau de conversão satisfatório. Nessa profundidade, o menor grau de conversão ocasionou o processo de degradação hidrolítica e erosão com exposição das partículas inorgânicas $(81,8 \%$ em peso de tamanho 0,1 a $10 \mu \mathrm{m})$, que conferiram a redução da microdureza nessa profundidade.

As propriedades mecânicas dos compósitos dependem do tipo, forma e porcentagem de partículas inorgânicas, composição monomérica, e da interface entre matriz e partícula (Fronza et al., 2015; Han et al., 2014; O’Neill et al., 2018; Sunbul et al., 2016). As partículas estão unidas quimicamente a matriz através de organosilanos bifuncionais, e podem se apresentar de forma diferente entre os diferentes materiais, para alguns compósitos podem ser pontos de ligações frágeis que favorecem a degradação da superfície (O’Neill et al., 2018). Portanto, uma correlação positiva entre dureza e percentual de partículas foi encontrada por HANAN et al, 2016. Neste estudo o compósito Z250 é o que apresenta maior percentual de partículas inorgânicas (81,8\% em peso), o que explica o melhor desempenho com relação a dureza de superfície em relação aos demais compósitos avaliados.

É possível ainda observar no presente estudo, que o tempo influenciou a dureza superficial nos dois meios avaliados para todos os materiais avaliados. Isso se deu, possivelmente, em virtude do ganho de água que pode prejudicar as propriedades dos compósitos resinosos e favorecer a degradação hidrolítica do material. A presença de componentes hidrófilos 
contribui acentuando a absorção do solvente. Monômeros como BIS-EMA, UDMA e TEGDMA possuem características hidrofílicas e estão presentes nos compósitos (Boaro et al., 2013; Sunbul et al., 2016).

No meio de imersão Etanol a 75\%, o compósito Filtek Z250 (Tabela 5), nas profundidades de 4 mm, nos períodos de 24 horas, apresentou redução dos valores médios de dureza com diferença estatística. Após o período de 180 dias houve redução dos valores de dureza com diferença significativa em todas as profundidades.

A densidade da rede de polímeros está relacionada com a permeabilidade do solvente. Uma rede rica em ligações intermoleculares diminui o volume de espaços livres, consequentemente reduzindo a penetração do solvente. Portanto, quanto mais reticulada a rede de polímero, menos a absorção de água e solubilidade é sofrida pelo material, e, por conseguinte, menores alterações de superfície são observadas (Zankuli et al., 2014).

As moléculas de água podem se acumular nos espaços vazios da rede de polímeros ou ligar-se a grupos iônicos específicos da cadeia de polímero (Alshali et al., 2013; Sunbul et al., 2016; Wei et al., 2011). Esse processo de difusão leva a degradação química, posteriormente os produtos de degradação são removidos da matriz, conduzindo a erosão do material com o tempo (Sunbul et al., 2016; Zankuli et al., 2014), o que provavelmente aconteceu com o compósito Z250 após o período armazenagem.

Neste estudo, quando o meio de armazenagem utilizado foi etanol a 75\% a redução da dureza foi pronunciada para todos os materiais. Os achados corroboram com os achados de HANAN et al, 2016, que observaram a redução de 50\% nos valores médios de dureza após imersão em álcool quando comparados com a água destilada, provavelmente esse resultado seja em virtude das diferenças nos parâmetros de solubilidade.

O compósito Tetric N-Ceram (Tabelas 2 e 3), apresentou menor dureza, com diferença estatística nos períodos e profundidades avaliadas, quando comparado aos compósitos Filtek Z250 e Filtek Bulk Fill. O Tetric N-Ceram é um compósito nanohíbrido com partículas inorgânicas de sílica, alumina e bário, com tamanho de 0,04 a 2,2 $\mu \mathrm{m}$, e percentual de $78 \% \mathrm{em}$ peso, sendo 17\% pré polimerizadas (Fronza et al., 2015; Han et al., 2014). A presença de partículas pré polimerizadas provavelmente influenciou o comportamento desse material nas condições de teste aqui observadas. Apesar de possuir na composição um alto percentual de partículas e a adição do fotoiniciador Ivocerin, mais reativo e derivado do dibenzoílo germânio associado a canforoquinona/sistema iniciador amino, muitos estudos tem demonstrado redução das propriedades mecânicas do compósito.

Pré-polímeros podem resultar em propriedades mecânicas reduzidas. Provavelmente, a presença dessas partículas influenciou a menor dureza demonstrada por este material no presente estudo, como resultado do processo de degradação hidrolítica e consequente erosão da matriz resinosa com exposição das partículas inorgânicas e degradação a partir da interface entre matriz e partícula (Fronza et al., 2015; O’Neill et al., 2018; Sunbul et al., 2016; Yap et al., 2016). Nos compósitos que possuem partículas pré polimerizadas, por existir poucas duplas ligações disponíveis na superfície dessas partículas, a união entre a matriz e as partículas pré-polimerizadas podem ser frágeis e resultar em falhas na interface favorecendo a degradação da superfície, o que provavelmente explica o pior desempenho desse compósito em relação aos outros analisados nesse estudo (Fronza et al., 2015; O’Neill et al., 2018).

O compósito Tetric N-Ceram, no meio de imersão Etanol 75\% (Tabela 5), demonstrou diferença significativa com o aumento da profundidade apenas em $4 \mathrm{~mm}$ no período de 24 horas. Nas profundidades de $4 \mathrm{~mm}$ houve aumento significativo no período de 180 dias de armazenagem, quando comparados a análise de 24 horas e 90 dias. Provavelmente, para este compósito, após os períodos de armazenagem e, com o processo de degradação hidrolítica e erosão da superfície por lixiviação dos componentes da matriz resinosa e das partículas pré-polimerizadas, houve a exposição das partículas inorgânicas, e, por conseguinte o aumento significativo na dureza por artefato da técnica, pois na verdade em algumas regiões possivelmente se mediu em parte a dureza das partículas inorgânicas expostas. 
Os valores de dureza estão relacionados ao conteúdo e tipo de partículas (Fronza et al., 2015; Sunbul et al., 2016; Yap et al., 2016), o compósito Filtek Bulk Fill é nanoparticulado de alta viscosidade com partículas de vidro fluoreto de ytérbio, cerâmica silanizada, sílica e zircônia $(4 \mathrm{~nm}$ a $0,1 \mu \mathrm{m})$, e percentual de $76,5 \%$ em peso. Este compósito apresentou comportamento intermediário com relação aos compósitos microhíbrido Filtek Z250 e, do tipo Bulk Fill Tetric N Ceram. O compósito Filtek Bulk Fill, no meio de imersão Etanol 75\% (Tabela 4), não apresentou diferença significativa na dureza nas profundidades e nos períodos de armazenagem. Este comportamento deve-se provavelmente ao fato de conter em sua composição alto conteúdo de partícula inorgânica $(76,5 \%)$, as nanopartículas, que favorecem a exposição de superfície mais regular, que se aproximam das características da matriz resinosa circunvizinha (O’Neill et al., 2018), mesmo após o processo de degradação hidrolítica e erosão observada ao longo do tempo, que não ocasionou grandes alterações com relação aos valores médios de dureza superficial.

É possível observar nas Tabelas 4 e 5 que a profundidade de polimerização influenciou a redução da microdureza nos dois meios avaliados, e nos materiais avaliados. $\mathrm{O}$ grau de polimerização afeta diretamente a microdureza, pois quanto maior $\mathrm{o}$ grau de conversão das ligações de carbono, maior o valor de dureza do compósito, por isso os corpos de provas dos compósitos analisados apresentam diferentes valores de dureza no topo e base (Júnior, 2002).

Diante da grande profundidade de polimerização deste material surge o questionamento quanto ao grau de polimerização, visto que a polimerização insuficiente compromete a qualidade da restauração com redução da durabilidade e eficiência do procedimento restaurador e das propriedades do compósito (Bucuta \& Ilie, 2014).

A redução gradual da microdureza superficial sugere uma diminuição no grau de conversão dos compósitos com o aumento da distância a partir da superfície irradiada, bem como a sua irradiância. As variações na profundidade de polimerização entre compósitos estudados podem ser inicialmente atribuídas à dispersão da luz nas interfaces de partículas e absorção de luz por fotoiniciadores que reduzem a penetração de luz e assim, também o grau de conversão de monômeros, que é determinada pela irradiação de luz em profundidade (Alrahlah et al., 2014).

\section{Conclusão}

De acordo com este estudo, concluiu-se que a profundidade de polimerização, tempo de armazenagem e o meio de imersão exerceu influência nos valores médios de dureza superficial dos compósitos, com a diminuição da dureza nos compósitos analisados, exceto no compósito Tetric N-Ceram, pois houve um aumento significativo de sua dureza com o decorrer do tempo, devido a características de sua composição. Além disso, a profundidade de polimerização influenciou a redução da microdureza superficial dos compósitos avaliados, de forma pronunciada para o compósito Filtek Z250, seguido do Tetric N Ceram. O meio de armazenagem influenciou a microdureza superficial, sendo mais pronunciado para o meio de armazenagem solução de Etanol a 75\%.

\section{Referências}

Alrahlah, A., Silikas, N., \& Watts, D. C. (2014). Post-cure depth of cure of bulk fill dental resin-composites. Dental Materials, 30(2), $149-154$.

Alshali, R. Z., Silikas, N., \& Satterthwaite, J. D. (2013). Degree of conversion of bulk-fill compared to conventional resin-composites at two time intervals. Dental Materials, 29(9), 213-217.

Assis, A. A. D. E. (2015). Desenvolvimento de um durômetro portátil para madeiras com o uso de um transdutor de deslocamento. Tese de Doutorado (Universidade Estadual Paulista)

Bicalho, A. A. et al. (2014). Incremental filling technique and composite material-Part II: Shrinkage and shrinkage stresses. Operative Dentistry, 39(2), 83-92.

Boaro, L. C. et al. (2013). Sorption, solubility, shrinkage and mechanical properties of "low-shrinkage" commercial resin composites. Dental Materials, 29(4), $398-404$.

Bucuta, S., \& Ilie, N. (2014). Light transmittance and micro-mechanical properties of bulk fill vs. conventional resin based composites. Clinical Oral 
Research, Society and Development, v. 10, n. 11, e183101119262, 2021

(CC BY 4.0) | ISSN 2525-3409 | DOI: http://dx.doi.org/10.33448/rsd-v10i4.19262

Investigations, 18(8), 1991-2000.

Campos, E. A. et al. (2014). Marginal adaptation of class II cavities restored with bulk-fill composites. Journal of Dentistry, 42(5), 575-581.

da Silveira, R. R. et al. (2012). Análise comparativa da microdureza superficial e profunda entre uma resina composta microhíbrida e uma resina composta de nanopartículas. Pesquisa Brasileira Em Odontopediatria e Clinica Integrada, 12(4), 529-534.

El-Damanhoury, H. M., \& Platt, J. A. (2014). Polymerization shrinkage stress kinetics and related properties of bulk-fill resin composites. Operative Dentistry, 39(4), 374-382.

El-Safty, S., Silikas, N., \& Watts, D. C. (2012). Creep deformation of restorative resin-composites intended for bulk-fill placement. Dental Materials, 28(8), 928-935.

Ferracane, J. L. (2011). Resin composite - State of the art. Dental Materials, 27(1), 29-38.

Fronza, B. M. et al. (2015). Monomer conversion, microhardness, internal marginal adaptation, and shrinkage stress of bulk-fill resin composites. Dental Materials, 31(12), 1542-1551.

Han, J. M. et al. (2014). Abrasive wear and surface roughness of contemporary dental composite resin. Dental Materials Journal, 33(6), 725-732.

Ibarra, E. T. et al. (2015). Physical properties of a new sonically placed composite resin restorative material. General Dentistry, 63(3), 51-56.

Júnior, E. A. de A. (2002). Influência da distância de uma fonte de luz halógena na microdureza superficial da resina composta. Dissertação de Mestrado (Universidade Estadual Paulista)

Leite, R. B. et al. (2018). Efeito de diferentes colutórios sobre a microdureza de resinas compostas fotopolimerizáveis. Revista de Odontologia Da UNESP, $47(3), 125-130$

O'Neill, C. et al. (2018). Effect of tooth brushing on gloss retention and surface roughness of five bulk-fill resin composites. Journal of Esthetic and Restorative Dentistry, 30(1), 59-69.

Ribeiro, R. A. D. O. et al. (2017). Propriedades das resinas bulk fill: uma revisão de literatura. Revista Odontologia Clínico-Científica, 16(2), 93-97.

Sunbul, H. Al, Silikas, N., \& Watts, D. C. (2016). Surface and bulk properties of dental resin- composites after solvent storage. Dental Materials, 32(8), 987997.

Tiba, A. et al. (2013). A Laboratory Evaluation of Bulk-Fill Versus Traditional Multi-Increment-Fill Resin-Based Composites. Journal of the American Dental Association, 144(10), 1182-1183.

Wei, Y. J. et al. (2011). Diffusion and concurrent solubility of self-adhering and new resin-matrix composites during water sorption/desorption cycles. Dental Materials, 27(2), 197-205.

Yap, A. U. J., Pandya, M., \& Toh, W. S. (2016). Depth of cure of contemporary bulk-fill resin-based composites. Dental Materials Journal, 35(3), 503-510.

Zankuli, M. A., Devlin, H., \& Silikas, N. (2014). Water sorption and solubility of core build-up materials. Dental Materials, 30(12), 324-329. 